\title{
Low fasting methionine concentration as a novel risk factor for recurrent venous thrombosis
}

\author{
Miranda B. A. J. Keijzer', Martin den Heijer', 2, George F. Borm², Henk J. Blom³, Stein Emil Vollset', \\ Ad R. M. M. Hermus', Per M. Ueland ${ }^{4}$ \\ 'Department of Endocrinology, ${ }^{2}$ Department of Epidemiology and Biostatistics, ${ }^{3}$ Laboratory of Paediatrics and Neurology, Radboud University \\ Nijmegen Medical Centre, Nijmegen, The Netherlands; ${ }^{4}$ Locus for Homocysteine and Related Vitamins, University of Bergen, Bergen, Norway
}

\section{Summary}

Hyperhomocysteinemia is a risk factor for venous thrombosis, but the underlying mechanism is unclear. If the thiol-group of homocysteine interferes with components of the clotting system, we expect that high cysteine will be also a risk factor for venous thrombosis. If high homocysteine reflects a disturbed methyl-group donation by S-adenosylmethionine, we expect that low methionine will be a risk factor for thrombosis. We performed a case-control study in 185 patients with recurrent venous thrombosis and in 500 control subjects.

We determined methionine, homocysteine, cysteine and assessed the associated thrombotic risk. Low fasting methionine was associated with an increased risk on recurrent venous

\section{Keywords}

Methionine, homocysteine, cysteine, venous thrombosis thrombosis $\left[\mathrm{OR}_{\text {bottom vs. top quartile }}=3.3(95 \% \mathrm{Cl}\right.$ I.9-5.7)]. Low methionine remained a risk factor $\left[\mathrm{OR}_{\text {bottom vs. top quartile }}=3.5\right.$ $(95 \% \mathrm{Cl} 2.0-6.0)]$ after adjusting for homocysteine and cysteine, whereas the thrombotic risk for homocysteine was lost [OR= I.0 (95\% Cl 0.6-I.9)] after adjustment. Cysteine yielded a highest odds ratio of $2 . I_{\text {top vs. bottom quartile }}(95 \% \mathrm{Cl} I .0-4.0)$ after adjustment. In conclusion, we found that low fasting methionine is a risk factor for recurrent venous thrombosis. This risk association was stronger for methionine than for homocysteine or cysteine.This supports the hypothesis that impaired methylation may be involved in the pathogenesis of venous thrombosis.

\section{Introduction}

Hyperhomocysteinemia is associated with the occurrence of arterial vascular disease and venous thrombosis (1-2). Several mechanisms have been suggested to explain the relationship between homocysteine and thrombotic disease. One hypothesis is that the thiol-group of homocysteine interferes with components of the clotting system (3-6). If this is true, it could be expected that other thiol-containing compounds, like cysteine, which has a close structural similarity to homocysteine, also have thrombogenic properties. Cysteine could therefore also be a risk factor for thrombosis, whereas methionine, which does not have a thiolgroup, would not be expected to be associated with an increased risk of thrombosis.

Another hypothesis focuses on a main purpose of the homocysteine metabolism, namely the donation of a methyl group by S-adenosylmethionine (AdoMet) (Fig. 1), which is used in many cellular methyltransferase processes, including methylation of DNA, RNA, lipids and proteins. A shortage of methionine leading to low AdoMet levels could influence several of the mentioned methylation processes, for instance DNA methylation (7), which in turn could contribute to the pathological cascade leading to venous thrombosis. If this hypothesis is true, hyperhomocysteinemia is merely a marker of an impaired metabolism of methyl groups, and one could expect that other metabolites that reflect this process, for instance low methionine, could also be risk factors for thrombosis. Hypercysteinemia, on the other hand, does not reflect this process, so it would not be expected to be a risk factor for thrombosis.

In order to determine which of the above hypotheses is more likely, we performed a case-control study and investigated the risk of recurrent venous thrombosis for metabolites and vitamins of the homocysteine metabolism, i.e. methionine, homocysteine, cysteine, betaine, plasma folate and red blood cell folate. We also 


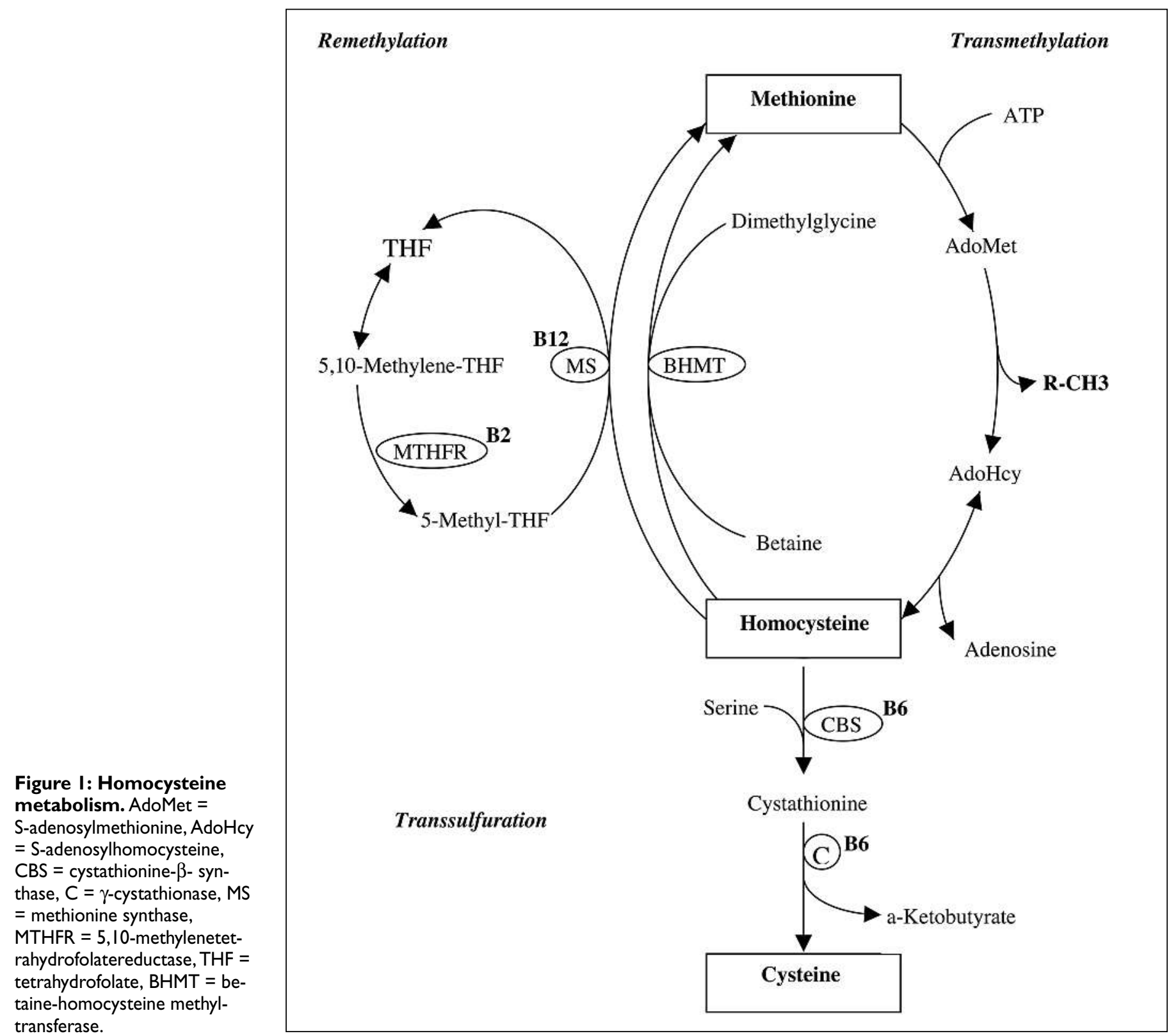

investigated whether risk associations were modified by compounds of the homocysteine metabolism or by the factor $\mathrm{V}$ Leiden or prothrombin $20210 \mathrm{G}>$ A mutation.

\section{Methods}

\section{Subjects}

For the current study, we used samples of a previously published case-control study $(8,9)$. In the Netherlands, virtually all patients with a history of venous thrombosis are registered at an anticoagulant clinic. We approached 473 patients who had had two or more episodes of venous thrombosis and invited them to take part in this study. Of those 473 patients, 185 patients (39\%) agreed to participate.

Through a general practice in The Hague, 2,812 possible control subjects between 20 and 90 years of age were invited by letter to participate in a study on risk factors for cardiovascular disease. Five hundred ninety-one $(21 \%)$ responded positively and subsequently received additional information by mail, after which 532 subjects agreed to participate. Of those 532 subjects, 10 as yet refused because of psychiatric problems (1), myocardial infarction (1), back pain (1) and undefined reasons (7). Of the 521 subjects left, 18 did not show up for blood sample collection, one subject refused venipuncture and for two subjects venipuncture was not possible. This left 500 control subjects who participated in the study. The only exclusion criterion was pregnancy.

The study was approved by the ethics committee of the Leyenburg Hospital, and informed consent was obtained from all participants. 


\section{Methods}

Because amino acid levels are influenced by diet, blood samples of all 185 patients and 500 control subjects were collected after overnight fasting. The samples were obtained from the antecubital vein in 4.5-ml EDTA vacuum glass tubes. They were used to determine the plasma concentrations of methionine, homocysteine, cysteine, folate, vitamin B12, vitamin B6 and vitamin B2 (riboflavin) and for detecting the methylenetetrahydrofolatereductase (MTHFR) 677C $>$ T, factor V Leiden $(1691 \mathrm{G}>\mathrm{A})$ and prothrombin $20210 \mathrm{G}>\mathrm{A}$ polymorphism.

The blood samples for plasma determinations were immediately placed on ice and centrifuged at $3,500 \mathrm{~g}$ for $5 \mathrm{~min}$ within two hours. The plasma was separated and stored at $-20^{\circ} \mathrm{C}$ until analysis. Total cysteine and total homocysteine concentrations in plasma were measured by an automated high-performance liquid chromatography method with fluorescent detection, essentially according to the method by Fiskerstrand et al. (10) which was slightly modified (11). The inter- and intra-run coefficient of variation was less than $6.5 \%$ for homocysteine concentrations (11) and less than 5\% for cysteine levels (12). Concentrations of methionine in plasma were determined by a modification of a method based on normal phase liquid chromatography- tandem mass spectrometry (13). Because methionine is oxidised during storage, we calculated the total methionine concentration as the sum of methionine and methionine sulfoxide. The inter- and intra-run coefficient of variation for methionine levels was about $5 \%$. Folate and vitamin B12 concentrations were measured in plasma samples stored at $-70^{\circ} \mathrm{C}$ with the Dualcount SPNB (solid phase no boil) Radioassay (Diagnostic Product Corporation, Los Angeles, CA, USA). Vitamin B6 (sum of pyridoxal 5'-phosphate and pyridoxal) and vitamin B2 were measured with liquid chromatography - tandem mass spectometry (14). MTHFR $677 \mathrm{C}>\mathrm{T}$, factor V Leiden and prothrombin $20210 \mathrm{G}>\mathrm{A}$ genotyping was performed as previously described (9).

\section{Statistical analysis}

Because we used data of a previously performed case-control study we calculated the "smallest detectable relative risk" (alpha 0.05 and power 0.8 ), which was 1.67 for an odds ratio (OR) above 1. Group characteristics are given as medians and ranges.

To investigate a possible dose-response relationship, we used the following logistic regression models: First, a univariate analysis was carried out for each of the variables methionine, homocysteine and cysteine. Second, each analysis was repeated with the additional covariables sex and age. Third, the variables methionine, homocysteine, cysteine, age and sex were all included and evaluated in one multivariate model. In an additional analysis, second order terms were included in the models to check for possible non-linearity and interactions (effect modification). We calculated $95 \%$ confidence intervals $(95 \% \mathrm{CI})$ for the OR.

For easy interpretation of the results, the OR could be interpreted as the relative risk to develop recurrent venous thrombosis for quartiles of the continuous variables. We used either the top quartile or the bottom quartile as a reference group. Because we did a case-control study in patients with recurrent venous thrombosis, the OR might be higher than expected for first-time venous thrombosis.

To evaluate a possible effect modification we calculated the relative risks for combinations of quartiles. For the combination of methionine and homocysteine, we used subjects with low homocysteine and high methionine as a reference group. For the combination of methionine with cysteine, we used subjects with low cysteine and high methionine as a reference group.

Furthermore, we investigated the joint effects for methionine in combination with the factor $\mathrm{V}$ Leiden, prothrombin 20210G $>$ A and MTHFR 677C $>$ T mutation. When the number of homozygote genotype variants was too small, as in the case of factor V Leiden, heterozygotes and homozygotes were taken together. We used subjects that had high methionine levels (top quartile) and wildtype factor $\mathrm{V}$ Leiden, prothrombin 20210G $>\mathrm{A}$ or wildtype MTHFR $677 \mathrm{C}>\mathrm{T}$ as a reference group.

We also investigated the risk of recurrent venous thrombosis associated with methionine concentrations below the median for subgroups of the MTHFR $677 \mathrm{C}>$ T mutation. Subjects with a methionine concentration above the median were the reference group.

\section{Results}

Baseline characteristics are shown in Figure 1. There was no significant correlation in control subjects between methionine and homocysteine concentration $(\mathrm{r}=-0.02 ; \mathrm{p}=0.72)$ and a weak correlation between methionine and cysteine concentration $(\mathrm{r}=$ $-0.10 ; \mathrm{p}=0.03)$. Homocysteine and cysteine levels were moderately correlated $(\mathrm{r}=0.329 ; \mathrm{p}<0.001)$.

\section{Univariate analysis}

We found methionine to be associated with a gradual increased risk ( $\mathrm{P}$ for trend $=<0.001$ ) of recurrent venous thrombosis with an age and sex-adjusted OR of 3.3 (95\%CI 1.9-5.7) for a methionine concentration below $20.6 \mu \mathrm{M}$ (Table 1). The thrombotic risk associated with homocysteine was also dose-dependent $(\mathrm{P}$ for trend $=0.01)$, with a highest adjusted risk of $1.4(95 \% \mathrm{CI}$ $0.8-2.4$ ) for the top quartile (Table 1). We also calculated the risk of recurrent venous thrombosis for subjects with a homocysteine concentration above the $90^{\text {th }}$ percentile, which yielded an adjusted (age and sex) OR of 1.7 (95\% CI 1.1 to 2.8), which is in line with previously published results $(8,9)$. For cysteine the dose response relationship was attenuated $(\mathrm{P}$ for trend $=0.3$ ) after adjusting for age and sex with a highest odds ratio of $1.9(95 \% \mathrm{CI}$ $1.0-3.4)$ for the top quartile.

\section{Multivariate analysis}

When the relative risks associated with quartiles of methionine, homocysteine and cysteine levels were not only adjusted for age and sex but also for each other, low methionine remained associated with an increased risk of venous thrombosis $[\mathrm{OR}=3.5$ (95\% CI 2.0-6.0)] whereas the thrombotic risk associated with homocysteine levels was lost for every of the upper three quartiles [OR=1.0 (95\% CI 0.6-1.9)] (Table 2). Cysteine yielded a highest OR of 2.1 (95\%CI 1.0-4.0) for the top quartile. 
Keijzer et al. Low methionine as a risk factor for venous thrombosis

Table I: Baseline characteristics of patients with recurrent venous thrombosis cases and control subjects*.

\begin{tabular}{|c|c|c|c|}
\hline Variable & Cases $(n=185)$ & Controls $(n=500)$ & P-value \\
\hline Age (year) & $62(42-79)$ & 50 (34 to 69$)$ & $<0.01$ \\
\hline Sex (men) & $94(5 \mid \%)$ & $208(42 \%)$ & 0.03 \\
\hline Post-menopausal women & $64(35 \%)$ & $138(28 \%)$ & $<0.01$ \\
\hline Time between Ist event and study (years) & $17(1-58)$ & - & \\
\hline Time between last event and study (years) & $7(\mathrm{I}-30)$ & - & \\
\hline Smoking (yes/no) & $61(33 \%)$ & $167(34 \%)$ & 0.9 \\
\hline B-Vitamin use (yes/no) & $23(12 \%)$ & $76(15 \%)$ & 0.9 \\
\hline Creatinine $(\mu \mathrm{M})$ & $8 \mid(6|-| \mid 2)$ & $74(55-99)$ & $<0.01$ \\
\hline Folate (nM) & I3.5 (7.7-23.5) & $12.7(7.0-23.6)$ & 0.2 \\
\hline Vitamin BI2 (pM) & $24 I(118-466)$ & $217(125-389)$ & 0.04 \\
\hline Vitamin B6 (nM) & $25.8(12.7-55.7)$ & $27.6(15.7-54.8)$ & 0.03 \\
\hline Vitamin B2 (nM) & $10.2(4.9-37.1)$ & $9.1(4.0-25.9)$ & 0.07 \\
\hline $\begin{array}{l}\text { FactorV Leiden (MAF) } \dagger \\
\text { - wildtype } \\
\text { - heterozygotes } \\
\text { - homozygotes }\end{array}$ & $\begin{array}{c}14.6 \% \\
124 \\
44 \\
3\end{array}$ & $\begin{array}{c}3.3 \% \\
438 \\
31 \\
0\end{array}$ & $<0.01$ \\
\hline $\begin{array}{l}\text { Prothrombin G20210A (MAF) } \dagger \\
\text { - wildtype } \\
\text { - heterozygotes }\end{array}$ & $\begin{array}{l}2.9 \% \\
10 \\
162\end{array}$ & $\begin{array}{l}1.6 \% \\
10 \\
162\end{array}$ & 0.12 \\
\hline $\begin{array}{l}\text { MTHFR C677T (MAF)† } \\
\text { - wildtype } \\
\text { - heterozygotes } \\
\text { - homozygotes }\end{array}$ & $\begin{array}{l}33.3 \% \\
75 \\
94 \\
14\end{array}$ & $\begin{array}{l}29.1 \% \\
247 \\
191 \\
45\end{array}$ & 0.21 \\
\hline
\end{tabular}

\section{Effect modification}

We calculated the OR for quartiles of methionine levels in combination with quartiles of homocysteine (Table 3) or cysteine concentrations (data not shown). The risk on recurrent venous thrombosis remained high in the bottom quartile of fasting methionine concentrations independent of homocysteine (Table 3) or cysteine (data not shown).

We repeated the analyses with adjustment for vitamin intake, smoking and blood levels of plasma folate, vitamin B12, vitamin B6 and vitamin B2. We found that low methionine is a risk factor for recurrent venous thrombosis independent of these variables (data not shown).

Next, we investigated a possible interaction between methionine and factor V Leiden or prothrombin $20210 \mathrm{G}>\mathrm{A}$, i.e. well-established genetic risk factors for venous thrombosis. The same analysis was performed for the MTHFR $677 \mathrm{C}>\mathrm{T}$ mutation. The combination of low methionine concentration (bottom quartile) and factor V Leiden yielded an adjusted (age and sex) OR of 15.9 ( $95 \%$ CI 5.5 to 45.9 ). In comparison, the OR for subjects with low methionine levels and no factor V Leiden was $3.9(95 \% \mathrm{CI} 2.0$ to 7.7) and it was $9.9(95 \% \mathrm{CI} 3.1$ to 32.0$)$ for subjects with factor $\mathrm{V}$ Leiden and high methionine levels (top quartile). However, because of the wide confidence interval no conclusive interpretations could be made about a possible biological interaction. No clear interaction was found between low methionine and the prothrombin 20210GA mutation or the MTHFR 677TT mutation.
Because the MTHFR 677TT genotype is a modest risk factor for venous thrombosis and is associated with elevated homocysteine levels, we performed an analysis to investigate whether the thrombotic risk associated with methionine levels below the median differed according to MTHFR genotype. Median methionine levels were similar in control subjects with the MTHFR 677TT genotype [23.6 $\mu \mathrm{M}$ (range 13.6 to 58.4 )] and the MTHFR $677 \mathrm{CC}$ genotype [24.0 $\mu \mathrm{M}$ (range 16.7 to 37.6 )]. The highest risk association for a methionine concentration below the median was found in subjects with the MTHFR 677TT genotype, yielding an odds ratio of $3.0(95 \% \mathrm{CI} 0.7-12.6)$. For the MTHFR $677 \mathrm{CC}$ group we found an OR of $2.0(95 \% \mathrm{CI} 1.1-3.5)$ for the risk of recurrent venous thrombosis associated with methionine levels below the median. However, because of the wide confidence intervals no conclusive interpretations can be draw.

\section{Discussion}

The main finding in this study is that low fasting methionine levels $(<20.6 \mu \mathrm{M})$ are associated with a 3.3 -fold $(95 \%$ CI 1.9-5.7) increased risk of recurrent venous thrombosis. In all analyses, whether multivariate or univariate, a decrease in methionine levels was associated with an increased risk of venous thrombosis. Furthermore, we found that methionine has a stronger risk association with recurrent venous thrombosis than homocysteine or cysteine. The thrombotic risk associated with methionine le- 
Keijzer et al. Low methionine as a risk factor for venous thrombosis

\begin{tabular}{|c|c|c|c|c|c|}
\hline Variables & Cases & Controls & $\begin{array}{l}\text { OR modell } \\
(95 \% \mathrm{Cl})\end{array}$ & $\begin{array}{l}\text { OR model2 } \\
(95 \% \mathrm{Cl})\end{array}$ & $\begin{array}{l}\text { OR model3 } \\
(95 \% \mathrm{Cl})\end{array}$ \\
\hline Plasma methionine & & & & $P<0.001^{\ddagger}$ & $P<0.001^{\ddagger}$ \\
\hline$<20.6(\mu \mathrm{M})$ & 62 & 108 & $2.9(1.7-4.7)$ & $3.3(1.9-5.7)$ & $3.5(2.0-6.0)$ \\
\hline $20.6-23.3(\mu \mathrm{M})$ & 48 & 123 & $1.9(1.2-3.3)$ & $2.0(1.2-3.5)$ & $2.1(1.2-3.6)$ \\
\hline $23.3-26.4(\mu \mathrm{M})$ & 46 & 124 & $1.8(1.1-3.1)$ & $1.8(1.0-3.1)$ & $1.9(1.1-3.3)$ \\
\hline$>26.4(\mu \mathrm{M})$ & 29 & 144 & $1.0^{\dagger}$ & $1.0^{\dagger}$ & $1.0^{\dagger}$ \\
\hline Plasma homocysteine & & & & $\mathrm{P}=0.0 \mathrm{I}^{\ddagger}$ & $P=0.8^{\ddagger}$ \\
\hline$<1 \mathrm{I} .3(\mu \mathrm{M})$ & 30 & 141 & $1.0^{\dagger}$ & $1.0^{\dagger}$ & $1.0^{+}$ \\
\hline II.3-13.4 ( $\mu \mathrm{M})$ & 40 & $|3|$ & $1.4(0.8-2.4)$ & I.I (0.7-2.0) & $1.0(0.6-1.8)$ \\
\hline $13.4-15.9(\mu \mathrm{M})$ & 49 & 122 & $1.9(1.1-3.2)$ & $1.2(0.7-2.0)$ & $1.0(0.5-1.7)$ \\
\hline$>15.9(\mu \mathrm{M})$ & 66 & 106 & $2.9(1.8-4.8)$ & $1.4(0.8-2.4)$ & $1.0(0.6-1.9)$ \\
\hline Plasma cysteine & & & & $P=0.3^{\ddagger}$ & $P=0.2^{\ddagger}$ \\
\hline$<198.4(\mu \mathrm{M})$ & 24 & 147 & $1.0^{\dagger}$ & $1.0^{\dagger}$ & $1.0^{+}$ \\
\hline $198.4-217.9(\mu \mathrm{M})$ & 42 & 129 & $2.0(I . I-3.5)$ & $1.5(0.8-2.7)$ & $1.6(0.9-2.9)$ \\
\hline $217.9-238.1(\mu \mathrm{M})$ & 45 & 126 & $2.1(1.3-3.8)$ & $1.3(0.7-2.3)$ & $1.4(0.7-2.5)$ \\
\hline$>238 . \mathrm{I}(\mu \mathrm{M})$ & 74 & 97 & $4.7(2.8-7.9)$ & $1.9(1.0-3.4)$ & $2.1(1.0-4.0)$ \\
\hline
\end{tabular}

Table 2: Odds ratio's for recurrent venous thrombosis for quartiles of amino acid concentrations.

\begin{tabular}{|c|c|c|c|c|}
\hline \multicolumn{5}{|c|}{ Plasma methionine $(\mu \mathrm{M})$} \\
\hline & $<20.6$ & $20.6-23.3$ & $23.2-26.4$ & $>26.4$ \\
\hline \multicolumn{5}{|c|}{ Plasma homocysteine } \\
\hline$<11.3(\mu \mathrm{M})$ & $6.7(1.7-27.3)$ & $4.2(1.0-17.8)$ & $3.0(0.7-12.6)$ & $1.0^{\dagger}$ \\
\hline II.3-13.4 ( $\mu \mathrm{M})$ & $4.3(1.1-17.5)$ & $2.5(0.6-11.8)$ & $4.4(1.1-16.9)$ & $1.7(0.4-7.7)$ \\
\hline $13.4-15.9(\mu \mathrm{M})$ & $5.9(1.5-22.9)$ & $2.8(0.7-11.0)$ & $3.8(0.9-15.5)$ & $1.3(0.5-5.6)$ \\
\hline$>15.9(\mu \mathrm{M})$ & $6.2(1.6-24.2)$ & $3.9(1.0-15.5)$ & $1.6(0.4-6.9)$ & $1.8(0.5-7.5)$ \\
\hline
\end{tabular}

Table 3: Odds ratio's $(95 \% \mathrm{Cl})$ for the combination of quartiles of methionine and homocysteine concentrations. vels below the median was highest in subjects with the MTHFR 677TT genotype. The combination of low methionine and factor V Leiden yielded an OR of 15.9 (95\% CI 5.5-45.9). However, confidence intervals were too wide to make conclusive interpretations about a possible biological interaction from the latter observation. Cysteine yielded a highest odds ratio of $2.1(95 \% \mathrm{CI}$ 1.0-4.0) for the top quartile after adjustment.

The mechanisms by which hyperhomocysteinemia may cause thrombotic disease are poorly understood. Homocysteine is a sulfur-containing amino acid that can only be formed from methionine (Fig. 1). Methionine is a precursor of S-adenosylmethionine (AdoMet), which serves as a methyl donor in transmethylation reactions. Demethylation of AdoMet results in the formation of S-adenosylhomocysteine (AdoHcy), which is hydrolyzed to homocysteine and adenosine. Homocysteine is degraded via the transsulfuration pathway or salvaged via remethylation to methionine (15). Homocysteine can be remethylated by methionine synthase for which 5-methyltetrahydrofolate is a substrate and vitamin B12 is a cofactor. An alternative remethylation pathway is catalyzed by betaine-homocysteine methyltransferase (BHMT), which uses betaine as a methyldonor.

The first hypothesis we investigated involves an impaired methylation pathway resulting in a reduced availability of methyl groups for methylation reactions, which may have a possible role in the development of venous thrombosis (7). If this is true, homocysteine is just a marker, and other metabolites that reflect this process, for instance low methionine, could also be expected to confer an increased thrombotic risk. The finding that low methionine is associated with a three-fold increased risk of recurrent venous thrombosis and that it is a stronger risk factor than high homocysteine or high cysteine, is in line with this hypothesis.

The loss of thrombotic risk for homocysteine after adjustment for methionine and cysteine levels and the finding that methionine has a stronger risk association with recurrent venous thrombosis is of interest and could suggest that methionine is a better marker for risk assessment of recurrent venous thrombosis. Quéré et al. (16) also found low methionine to be stronger associated with venous thrombosis than hyperhomocysteinemia in 
a matched case-control study in 243 patients with venous thrombosis, with an OR of 2.4 (95\% CI 1.37-4.20) for methionine levels below the median in a multivariate analysis.

The MTHFR $677 \mathrm{C}>\mathrm{T}$ mutation also has been associated with an increased risk on venous thrombosis (2), which is thought to be due to elevation of homocysteine. We found that the risk of recurrent venous thrombosis associated with a methionine level below the median is highest in subjects with the MTHFR 677TT genotype, which in theory could also explain the association between MTHFR 677TT and venous thrombosis.

Because determinants of methionine levels are poorly studied, it is difficult to indicate causes underlying low methionine levels. It could be possible that a low plasma methionine level is the result of a low intake of methionine. Another explanation could be impaired remethylation of homocysteine to methionine. In subjects with homocystinuria severe MTHFR deficiency is often accompanied by low methionine levels (17). However, in our study we did not find significantly lower methionine levels in subjects with the MTHFR 677TT genotype, which causes a mildly reduced enzyme activity and increased thermolability (18).

The other hypothesis tested in our study was the role of the thiol-group of homocysteine (3-6). If the thiol-group of homocysteine is involved in thrombogenesis, it might be expected that cysteine, a sulfhydryl amino acid which is structurally very simi- lar to homocysteine, is also associated with an increased risk of thrombosis. We found no dose-response relationship between cysteine levels and the risk of venous thrombosis after adjustment for age and sex. However, high cysteine levels (top quartile) were associated with a two-fold increased thrombotic risk after adjusting for age and sex. With regard to this data we cannot draw solid conclusions whether cysteine is a risk factor for recurrent venous thrombosis. We found some other studies that investigated the relationship between elevated cysteine concentrations and cardiovascular disease (19-26). Only one study reported on a role of hypercysteinemia in venous thrombosis (20). In that study, hypercysteinemia $[\mathrm{OR}=2.9(95 \% \mathrm{CI} 1.1-7.8)]$ and hyperhomocysteinemia $[\mathrm{OR}=8.0(95 \% \mathrm{CI} 3.6-18.0)]$ were both risk factors for venous thrombosis; however, they did not adjust for methionine levels.

In conclusion, we found low fasting methionine to be a risk factor for recurrent venous thrombosis. This risk association was stronger for methionine than for homocysteine and cysteine. This observation supports the hypothesis that impaired methylation may play a role in the development of recurrent venous thrombosis in hyperhomocysteinemia.

\section{Acknowledgements}

Martin den Heijer is recipient of a VENI stipend (NWO) of the Netherlands Organisation for Scientific Research.

\section{References}

1. The Homocysteine Studies Collaboration. Homocysteine and risk of ischemic heart disease and stroke. J Am Med Assoc 2002; 288: 2015-22.

2. Den Heijer M, Lewington S, Clarke R. Homocysteine, MTHFR and risk of venous thrombosis: a metaanalysis of published epidemiological studies. Thromb Haemost 2005; 3: 292-9.

3. Majors AK, Sengupta S, Willard B, et al. Homocysteine binds to human plasma fibronectin and inhibits its interaction with fibrin. Arterioscler Thromb Vasc Biol 2002; 22: 1354-9.

4. Harpel PC, Chang VT, Borth W. Homocysteine and other sulfhydryl compounds enhance the binding of lipoprotein(a) to fibrin: A potential biochemical link between thrombosis, atherogenesis, and sulfhydryl compound metabolism. Proc Natl Acad Sci USA 1992; 89: 10193-7.

5. Undas A, Williams EB, Butenas S, et al. Homocysteine inhibits inactivation of factor Va by activated protein C. J Biol Chem 2001; 276: 4389-97.

6. Lauricella AM, Quintana IL, Kordich LC. Effects of homocysteine thiol group on fibrin networks: another possible mechanism of harm. Thromb Res 2002; 107: 75-9.

7. Castro R, Rivera I, Struys EA, Jansen EEW, Ravasco P, Camilo ME, Blom HJ, Jakobs C, Tavares de Almeida I. Increased homocysteine and S-Adenosylhomocysteine concentrations and DNA hypomethylation in vascular disease. Clin Chem 2003; 49: 1292-6. 8. Den Heijer M, Blom HJ, Gerrits WBJ, Rosendaal FR, Haak HL, Wijermans PW, Bos GMJ. Is hyperhomocysteinaemia a risk factor for recurrent venous thrombosis? Lancet 1995; 345: 882-5.

9. Keijzer MBAJ, den Heijer M, Blom HJ, et al. Interaction between hyperhomocysteinemia, mutated methylenetetrahydrofolate reductase (MTHFR) and in- herited thrombophilic factors in recurrent venous thrombosis. Thromb Haemost 2002; 88: 723-8. 10. Fiskerstrand T, Refsum $H$, Kvalheim G, et al. Homocysteine and other thiols in plasma and urine: automated determination and sample stability. Clin Chem 1993; 39: 263-71.

11. Te Poele-Pothoff MT, van den Berg M, Franken DG, et al. Three different methods for the determination of total homocysteine in plasma. Ann Clin Biochem 1995; 32: 218-20.

12. De Graaf-Hes A, Trijbels F, Blom HJ. New method for determining cystine in leucocytes and fibroblasts. Clin Chem 1999; 45: 2224-8.

13. Holm PI, Ueland PM, Kvalheim G, et al. Determination of choline, betaine, and dimethylglycine in plasma by a high-throughput method based on normal phase chromatography-tandem mass spectrometry. Clin Chem 2003; 49: 286-94.

14. Midttun O, Hustad S, Solheim E, et al. Multianalyte quantification of vitamin B6 and B2 species in the nanomolar range in human plasma by liquid chromatography-tandem mass spectrometry. Clin Chem 2005; 51: $1206-16$.

15. Selhub J, Miller JW. The pathogenesis of homocysteinemia: Interruption of the coordinate regulation by S-adenosylmethionine of the remethylation and transsulfuration of homocysteine. Am J Clin Nutr 1992; 55: 131-8.

16. Quéré I, Perneger TV, Zittoun J, et al. Red blood cell methylfolate and plasma homocysteine as risk factors for venous thromboembolism: a matched case-control study. Lancet 2002; 359: 747-52.

17. Fenton WA, Rosenblatt DS. Inherited Disorders of Folate and Cobalamin Transport and Metabolism. In: The Metabolic and Molecular Bases of Inherited Disease, $8^{\text {th }}$ ed., McGraw-Hill, New York 2001; 3897-933.

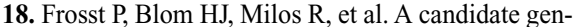
etic risk factor for vascular disease: A common mutation in methylenetetrahydofolate reductase. Nat Genet 1995; 10: 111-3.

19. Mills BJ, Weiss MM, Lang CA, et al. Blood glutathione and cysteine changes in cardiovascular disease. $\mathrm{J}$ Lab Clin Med 2000; 135: 396-401.

20. Marcucci R, Brunelli T, Giusti B, et al. The role of cysteine and homocysteine in venous and arterial thrombotic disease. Am J Clin Pathol 2001; 116: 56-60.

21. Jacob N, Bruckert E, Giral P, et al. Cysteine is a cardiovascular risk factor in hyperlipidemic patients. Atherosclerosis 1999; 146: 53-9.

22. Mansoor MA, Bergmark C, Svardal AM, et al. Redox status and protein binding of plasma homocysteine and other aminothiols in patients with early-onset peripheral vascular disease. Arterioscler Thromb Vasc Biol 1995; 15: 232-40.

23. Araki A, Sako Y, Fukushima Y, et al. Plasma sulfhydryl-containing amino acids in patients with cerebral infarction and in hypertensive subjects. Atherosclerosis 1989; 79: 139-46.

24. Özkan Y, Özkan E, Simsek B. Plasma total homocysteine and cysteine levels as cardiovascular risk factors in coronary heart disease. Intern J Cardiol 2002; 82: 269-77.

25. Verhoef P, Stampfer MJ, Buring JE, et al. Homocysteine metabolism and risk of myocardial infarction: Relation with vitamins $\mathrm{B}_{6}, \mathrm{~B}_{12}$, and folate. Am J Epidemiol 1996; 143: 845-59.

26. El-Khairy L, Ueland PM, Refsum H, et al. Plasma total cysteine as a risk factor for vascular disease: The European Concerted Action Project. Circulation 2001; 103: 2544-9. 